\title{
Long-Term Results of Radiofrequency Catheter Ablation in Non-ischemic Sustained Ventricular Tachycardia with Underlying Heart Disease

\author{
Nonuniform Arrhythmogenic Substrate \\ and Mode of Ablation
}

\author{
Masaomi Chinushi, MD, Yoshifusa Aizawa, MD, \\ Kouji OHHIRA, MD, Akira ABE, MD \\ and Akira ShIBATA, MD
}

\begin{abstract}
SUMmary
This study examined 12 VTs in 8 patients who underwent radiofrequency (RF) catheter ablation for ventricular tachycardia (VT) associated with non-ischemic underlying heart diseases, and who were followed-up for more than 24 months after ablation. The site of VT origin was determined to be within a narrow site (within $1.0 \times 1.0 \mathrm{~cm}$ ) in 5 VTs ( 4 patients), but VT originated from a wide origin (more than $1.0 \times 1.0 \mathrm{~cm}$ ) in the other 5 VTs $(3$ patients). The remaining patient had two macroreentrant VTs revolving around an anatomical obstacle in both the clockwise and counterclockwise directions. Two of 5 VTs originating from a narrow site were successfully ablated by 2-3 RF applications. In VT associated with a wide origin, two perpendicular linear RF lesions with $6.0 \pm 1.8 \mathrm{RF}$ applications were required to ablate the VT. Eight of the 12 VTs $(66.7 \%)$ were finally ablated by RF current (30-50 watts), and they did not recur during the follow-up period of $31.2 \pm 6.5$ months.

An excellent long-term outcome is expected, even in VT associated with non-ischemic underlying heart disease, if VT is successfully treated by RF ablation. (Jpn Heart J 1996; 37: 183-194)
\end{abstract}

Key words: RF ablation Non-ischemic VT Long-term outcome

\begin{abstract}
R ADIOFREQUENCY (RF) catheter ablation has been used for the treatment of ventricular tachycardia (VT), and excellent short-term results have been reported, especially in VT without underlying heart diseases ${ }^{1-3)}$ and in VT caused by coronary artery disease.,5) However, the indication and efficacy of RF ablation in VT associated with non-ischemic underlying heart diseases have not yet been established.
\end{abstract}

From the First Department of Internal Medicine, Niigata University School of Medicine, Niigata, Japan.

Address for correspondence: Masaomi Chinushi, MD, First Department of Internal Medicine, Nügata University School of Medicine, 1-754 Asahimachi, Niigata 951 Japan.

Received for publication October 16, 1995.

Accepted January 8, 1996. 
The purpose of this study was to assess the long-term results of RF ablation in VT associated with non-ischemic underlying heart diseases. The electrophysiological characteristics of these VTs were also assessed in relation to the response to RF ablation.

\section{Methods}

Subjects: RF ablation has been employed for the treatment of drug refractory ventricular tachyarrhythmia at our hospital since October 1991, and a total of 58 patients had undergone this therapy as of December 1994. Of these, 8 patients were selected for study. The criteria for patient selection were as follows: 1) they had recurrent episodes of symptomatic monomorphic sustained VT, 2) RF catheter ablation was employed for the treatment of VT, and the patients were followed-up for more than 24 months after ablation, 3) non-ischemic underlying heart diseases were diagnosed using conventional examination methods, including a two-dimensional Doppler echocardiogram, cardiac catheterization and/or magnetic resonance imaging. Ischemic heart disease patients were excluded based on clinical symptoms, 12-lead electrocardiogram, exercise stress test and coronary angiography.

The patients consisted of 6 males and 2 females, and age ranged from $16^{-}$

Table. Characteristics of Patients and VT

\begin{tabular}{|c|c|c|c|c|c|c|c|c|c|c|c|c|}
\hline \multirow[b]{2}{*}{ Case } & \multirow[b]{2}{*}{ UHD } & \multirow[b]{2}{*}{ VTCL } & \multirow[b]{2}{*}{$\begin{array}{c}\text { Mecha- } \\
\text { nism }\end{array}$} & \multirow[b]{2}{*}{ Origin } & \multirow[b]{2}{*}{ Extent } & \multirow[b]{2}{*}{$\begin{array}{c}\text { Target } \\
\text { Site }\end{array}$} & \multirow[b]{2}{*}{ LEG } & \multirow[b]{2}{*}{ Watts } & \multicolumn{2}{|c|}{ Attempt } & \multirow{2}{*}{ - Resuit } & \multirow{2}{*}{$\begin{array}{l}\text { Follow- } \\
\text { up }\end{array}$} \\
\hline & & & & & & & & & $\begin{array}{l}\text { Ineffec- } \\
\text { tive }\end{array}$ & $\begin{array}{c}\text { Effec- } \\
\text { tive }\end{array}$ & & \\
\hline 1. $53 \mathrm{M}$ & ARVD & $250 \mathrm{I}$ & non-reentry & RVOT & & $\begin{array}{c}\operatorname{EAS}(-30- \\
-40)\end{array}$ & 70 & 30 & 2 & 2 & success & 41 \\
\hline \multirow[t]{2}{*}{ 2. $17 \mathrm{M}$} & DORV & $260 \mathrm{r}$ & reentry & RVA & narrow & EAS $(-20)$ & 70 & 40 & 6 & 0 & failure & (35) \\
\hline & & $270 \mathrm{r}$ & reentry & LV sep & narrow & $\operatorname{EAS}(-35)$ & 90 & 40 & 4 & 0 & failure & \\
\hline 3. $36 \mathrm{M}$ & $\mathrm{DCM}$ & $300 \mathrm{n}$ & non-reentry & RVOT & wide & $\operatorname{EAS}(-50)$ & 60 & $40-50$ & 10 & 8 & success & 34 \\
\hline $4.51 \mathrm{~F}$ & DCM & $480 r$ & reentry & RVOT & wide & $\operatorname{SCZ}(-120)$ & 320 & $40-50$ & 19 & 7 & cess & 30 \\
\hline $5.21 \mathrm{~F}$ & DORV & $260 \mathrm{r}$ & reentry & RV sep & narrow & $\mathrm{SCZ}(-15)$ & $\mathrm{CA}$ & $40-50$ & 6 & 0 & failure & (28) \\
\hline \multirow[t]{3}{*}{ 6. $68 \mathrm{M}$} & DCM & $260 \mathrm{r}$ & reentry & LV & wide & SC7. $(-70)$ & 190 & $40-50$ & 3 & 5 & success & $(28)$ \\
\hline & & $300 \mathrm{r}$ & reentry & LV & wide & $\operatorname{EAS}(-30)$ & 140 & $40-50$ & 12 & 0 & failure & \\
\hline & & $280 \mathrm{r}$ & reentry & $\mathrm{LV}$ & wide & $\mathrm{SCZ}(-50)$ & 160 & $40-50$ & 1 & 4 & success & \\
\hline \multirow[t]{2}{*}{ 7. $16 \mathrm{M}$} & $\mathrm{T} / \mathrm{F}$ & $410 \mathrm{r}$ & reentry & RVOT & macro & SCZ $(-160)$ & 280 & 40 & 2 & 5 & success & 26 \\
\hline & & $430 \mathrm{r}$ & reentry & RVOT & macro & $\operatorname{SCZ}(-120)$ & 140 & 40 & 2 & 3 & success & \\
\hline $8.56 \mathrm{M}$ & ARVD & $230 \mathrm{r}$ & reentry & RVA & narrow & $\operatorname{EAS}(-40)$ & 140 & $40-50$ & 5 & 3 & success & 25 \\
\hline
\end{tabular}

ARVD = arrhythmogenic right ventricular dysplasia; $\mathrm{CA}=$ continuous activity; $\mathrm{DCM}=$ idiopathic dilated cardiomyopathy; DORV = double outlet of the right ventricle; $\mathrm{EAS}=$ earliest activation site (msec); $\mathrm{LEG}=$ duration of the electrogram (msec); LV = left ventricle; LV sep = septum of the left ventricle, macro = macroreentry; Origin = site of origin; RVA = apex of the right ventricle; RVOT = outflow tract of the right ventricle; $\mathrm{RV}$ sep = septum of the right ventricle; $\mathrm{SCZ}=$ slow conduction zone (msec); $\mathrm{T} / \mathrm{F}=$ tetralogy of Fallot, $\mathrm{UHD}=$ underlying heart disease; VTCL = cycle length of VT (msec); Other abbreviations are the same as in text. 
68 years (mean \pm SD: $39.8 \pm 20.0$ ) (Table). All patients had non-ischemic underlying heart disease: specifically idiopathic dilated cardiomyopathy (3 patients), post-operative congenital heart disease (3), and arrhythmogenic right ventricular dysplasia (2). Five patients had a single VT-QRS morphology, while the other 2 patients showed two different VT-QRS morphologies. In the remaining patient, 3 distinct VT-QRS morphologies were clinically confirmed. The mean cycle length of VT was $310 \pm 82 \mathrm{msec}$ (ranging from 230 to $480 \mathrm{msec}$ ).

The number of clinical episodes of symptomatic sustained VT prior to RF ablation was from 6 to 9 times in 3 patients, and more than 10 times in the other 5 patients. Before admission to our hospital, all patients complained of palpitations during the VT episodes, and 3 had a history of syncope. Conventional antiarrhythmic drug therapy (2-5 drugs) failed to prevent the recurrence and/or induction of VT. Three patients were prescribed amiodarone before RF ablation, but it was not effective at suppressing the VT.

Electrophysiologic Study: Informed consent was obtained from the patients after thoroughly explaining the procedure, results, and possible risks. Electrophysiologic studies were performed in the nonsedated and post-absorptive state. All antiarrhythmic drugs were discontinued at least 5 half-lives before the control study. Amiodarone was not administered before the control study. Three $6 \mathrm{~F}$ quadripolar catheters with interelectrode distances of $5 \mathrm{~mm}$ were positioned within the heart under fluoroscopic guidance. Stimulation was performed using the distal and third electrodes, and an intracardiac electrogram was recorded using the second and fourth electrodes of the same catheter. Otherwise, local electrograms were recorded using the distal two electrodes. Intracardiac electrograms were recorded on an ink-jet recorder (Siemens Elema Co. Ltd. Mingograf 7) with three surface leads (I, II, and V1) at a paper speed of either 100 or 200 $\mathrm{mm} / \mathrm{sec}$. The band-pass filter was set at 50 to $500 \mathrm{~Hz}$.

Protocol for VT Induction: Electrical stimuli were delivered at twice the late diastolic threshold of $2 \mathrm{msec}$ using a cardiac stimulator (Fukuda Denshi Co, Cardiac Stimulator BC02). Our standard stimulation protocol ${ }^{6,7)}$ consisted of 1 to 3 extrastimuli delivered after 8 basic stimuli at 2 different basic cycle lengths $(400$ and $600 \mathrm{msec}$, and rapid pacing at cycle lengths up to $286 \mathrm{msec}$. Stimulation was attempted at two sites on the right ventricle (apex and outflow tract). If this procedure did not induce VT, isoproterenol was infused to increase the sinus rate by 20 percent, and stimulation was repeated using the same method at two right ventricle sites and one left ventricle site.

Endocardial mapping and ablation site: The site of VT origin was determined as the earliest activation site during VT using a steerable 7 French quadripolar catheter with a 5-mm interelectrode distance (Mansfield, Boston Scientific International). When a local electrogram with an almost identical acti- 
vation time (the difference $<10 \mathrm{msec}$ ) was recorded from nearby multiple sites, VT was considered to have originated not from a point but from an area, and the area showing the same earliest isochronal presystolic electrocardiogram was determined ${ }^{87}$ During mapping, the interelectrode distance of the mapping catheter was used as a marker to estimate the distance, and the location of the catheter was carefully checked by bi-directional fluoroscopy.

If an abnormal local electrogram, such as mid-diastolic potential or continuous activity, was recorded during VT, rapid pacing was performed and the central common slow pathway of the reentry circuit was determined by the criteria described by Fontaine et al. ${ }^{9)}$ Pace-mapping during sinus rhythm ${ }^{10)}$ was also used to facilitate mapping of the site of VT origin, although the site of origin was not based on pace-mapping alone.

Ablation procedure: At RF ablation, antiarrhythmic drugs were used in 4 patients to slow the VT rate. RF currents were produced by a generator (HAT 200 OSYPKA $520 \mathrm{KHz}$ ), and delivered at 30-50 watts to the distal $4 \mathrm{~mm}$ tip of the electrode positioned on the target site. An external patch-electrode was placed on the back. Whether or not RF was delivered to a proper site was judged by observing the termination of VT within 10 seconds. ${ }^{2,8)}$ In such instances, the current was further delivered for 30 seconds. Otherwise, the current was discontinued at 10 seconds and the appropriate ablation site remapped. The current was turned off immediately if an impedance began to rise.

After ablation, intravenous administration of heparin sodium was continued for a day at a dosage of 10,000-15,000 units. Electrocardiograms were monitored until the patient was discharged. Serum creatinine kinase (CK) and CKMB were measured at 4-6 and 12-24 hours after ablation. A two-dimensional echocardiogram was recorded immediately and 1-2 weeks after the procedure.

Efficacy of RF ablation: The inducibility of VT was assessed at the end of the procedure and 2 weeks after ablation. Ablation was judged to be successful if VT was not induced after completion of the induction protocol. Complete disappearance of spontaneous sustained and non-sustained VT after ablation was also confirmed by electrocardiographic monitoring.

The suppression of VT induction with antiarrhythmic drugs which were ineffective before RF ablation was considered to be a clinical success.

Follow-up and evaluation of results: All patients were followed-up in our outpatient clinic at least once a month. Follow-up data were obtained directly from the patients. Since all patients had been aware of each episode of VT, VT recurrence was assessed on the basis of symptoms and Holter electrocardiograms.

\section{Definitions:}

1) Sustained VT was defined as VT that lasted for more than 30 seconds, or which required emergency termination within 30 seconds. Nonsustained VT 
was defined as VT lasting 30 seconds or shorter.

2) RF current was delivered during VT, and effective RF application was defined as that in which sustained VT was terminated by RF current within 10 seconds. Otherwise, RF application was defined as ineffective.

3) A VT origin determined to be within a site extending $1.0 \times 1.0 \mathrm{~cm}$ was defined as narrow origin. An origin extending more than $1.0 \times 1.0 \mathrm{~cm}$ was defined as a wide origin.

4) According to earlier reports, ${ }^{11,12)}$ transient entrainment was defined as follows: (1) Constant fusion during rapid pacing except for the last non-fused but captured beat that occurred at the paced cycle length in the surface electrogram or in the electrogram at the exit from the area of slow conduction. (2) A constant but different degree of fusion at different pacing rates. (3) Advancement of the local electrogram at the exit without a change in the morphology.

Statistical Analysis: Fisher's exact probability test was used to assess the differences between 2 groups. A p value of less than 0.05 was considered significant. Values are presented as the mean \pm SD.

\section{Results}

Characteristics of VT: Twelve monomorphic VTs $(5$ with a right bundle branch block and 7 with a left bundle branch block pattern) were induced in 8 patients (Table).

VT was reproducibly induced and terminated by programmed electrical stimulation, and transient entrainment was observed during 10 VTs (6 patients). The other two VTs occurred spontaneously during administration of isoproterenol. No VT was induced by the treadmill exercise test.

Site of VT Origin: The site of VT origin was determined either as the earliest activation site during VT $(n=6)$ or the central common slow pathway within the reentry circuit $(n=6)$.

VT origin was the outflow tract of the right ventricle in 5 VTs (4 patients), the inflow tract of the right ventricle in $2 \mathrm{VTs}$ (2 patients) and the free wall of the left ventricle in 3 VTs (one patient). VT arose from the right ventricular side of the intraventricular septum in one VT, and from the left ventricular side of the intraventricular septum in the other VT (Table).

In 5 VTs (4 patients), VT was considered to have originated from a narrow site, and the earliest activation site of the VT (4 VTs) or central common slow pathway in the reentry circuit was determined to be within the site extending 1.0 $\times 1.0 \mathrm{~cm}$. The 5 other VTs ( 3 patients) originated from a wide origin. In these VTs, isochronal presystolic local electrograms were obtained from an area greater than $2.0 \times 2.0 \mathrm{~cm}$, and the area seemed to be related to their occurrence 


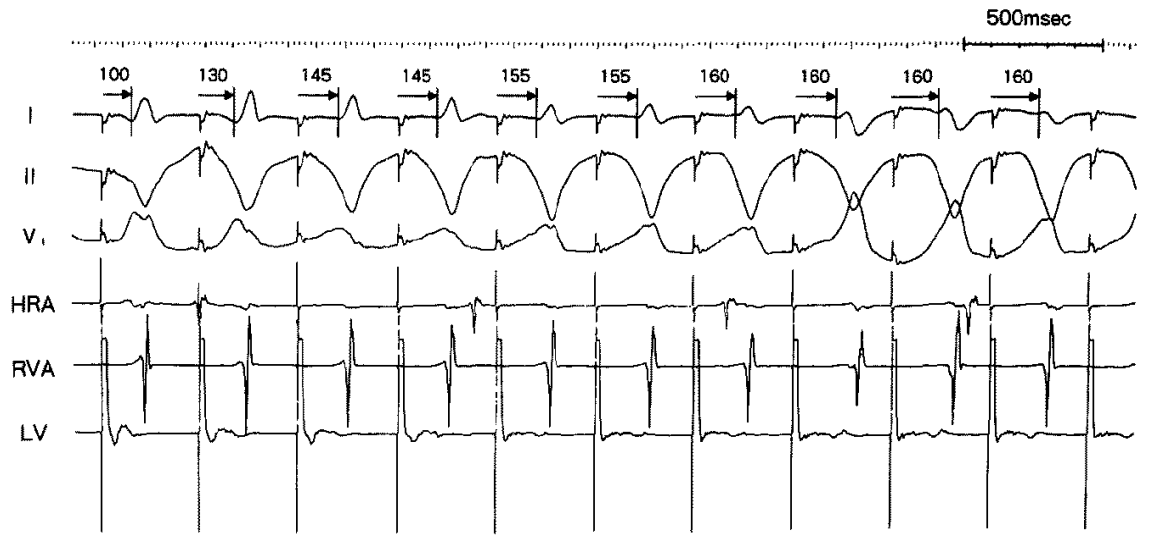

Figure 1. Constant pacing at arrhythmogenic area. During constant pacing within a wide arrhythmogenic area (Case 6), multiple paced-QRS morphologies were observed concomitant with progressively prolonged intervals (more than $100 \mathrm{msec}$ ) from the stimulus artifact to the onset of the QRS complex. Multiple slow pathways within the area seemed to best explain this finding. Some of the paced QRS morphologies were similar but not identical to the VT-QRS morphology. HRA = high right atrium; $\mathrm{RVA}=$ apex of the right ventricle; $L V=$ left ventricle.

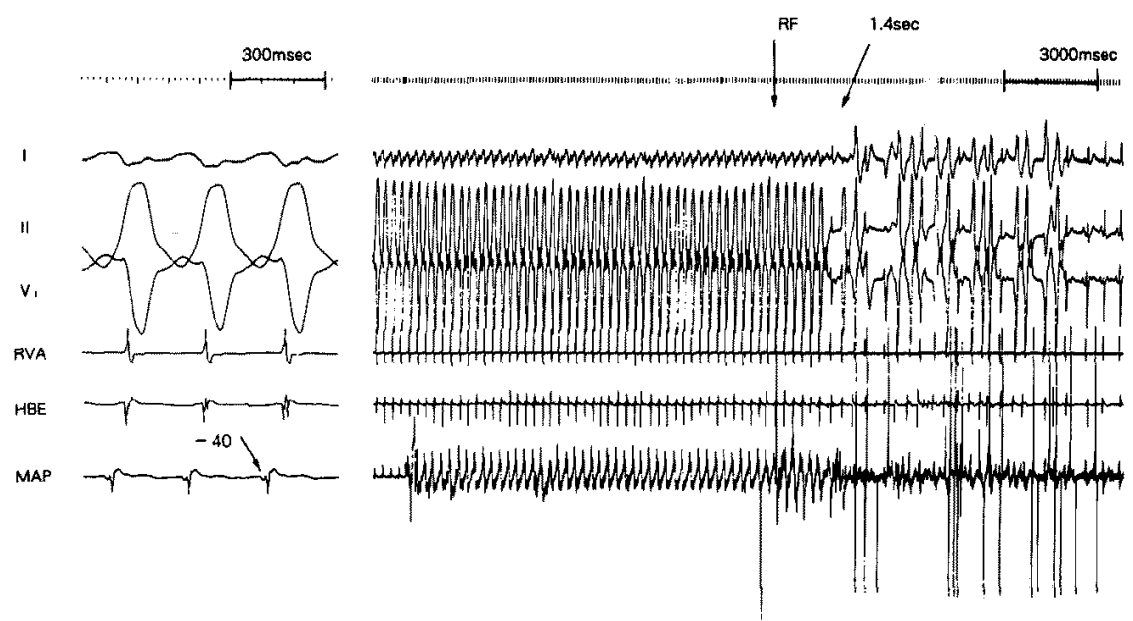

Figure 2. RF ablation for VT originating from a narrow site. This patient was a 53year-old man (Case 1). The local electrogram at the ablation site was $40 \mathrm{msec}$ before onset of the QRS complex. The VT was terminated by RF application and became non-inducible. $\mathrm{HBE}=$ His-bundle electrogram, $\mathrm{MAP}=$ mapping site. Other abbreviations are the same as in Figure 1.

and/or maintenance. The remaining 2 VTs were macroreentry revolving around the presumed operative scar in clockwise and counterclockwise directions as reported previously. ${ }^{13)}$ The best pacemapping was obtained from the site (or area) of VT origin, but multiple paced $\mathrm{QRS}$ morphologies concomitant with different long intervals (more than $40 \mathrm{msec})^{14,15)}$ from the stimulus artifact to the 


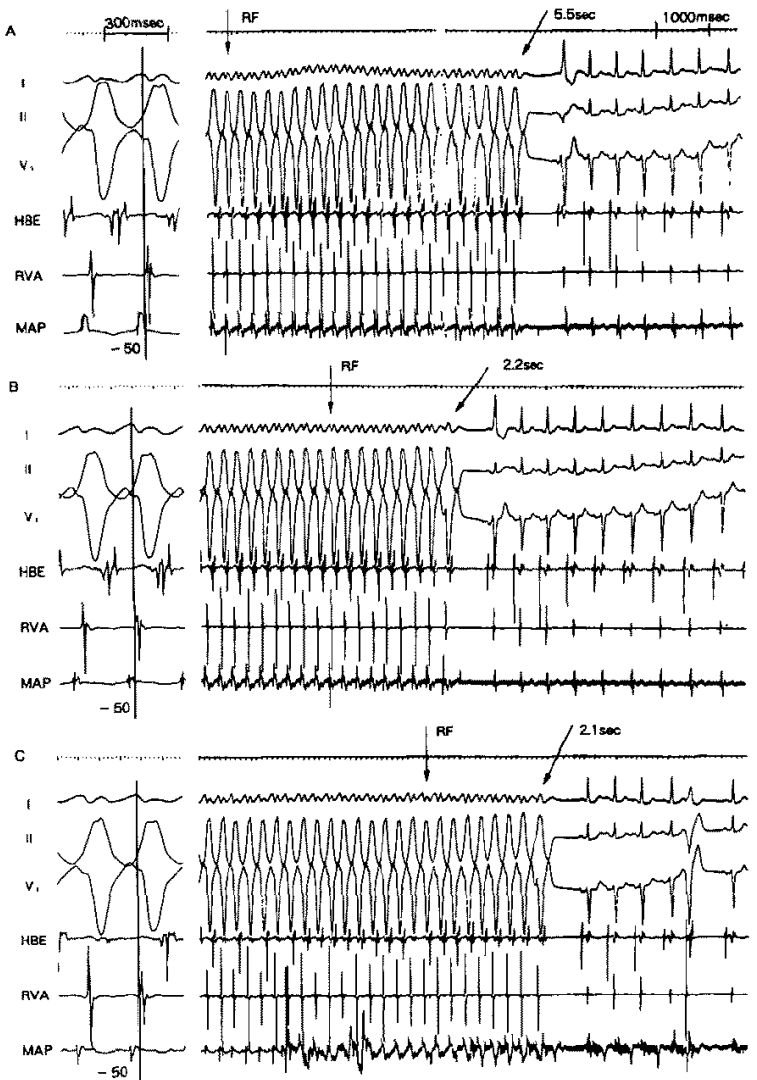

Figure 3. Endocardial mapping and RF ablation. This patient was a 36-year-old man (Case 3). As shown in panels $A$ to $C$, an arrhythmogenic area of $2.0 \times 2.0 \mathrm{~cm}$ was determined and each local electrogram from within this area was $50 \mathrm{msec}$ before onset of the QRS complex. However, the morphologies of each local electrogram were different. Ablation of each of these sites terminated the VT, although VT with the same morphology occurred until the area was covered by RF lesions in the form of 2 perpendicular lines. Abbreviations are the same as in Figures 1 and 2.

onset of the QRS complex were sometimes obtained from the arrhythmogenic site (Figure 1). These QRS morphologies were similar but not completely identical to those of the VTs, even at the site of successful ablation.

During VT, the local electrogram at the site of origin was found to be $63 \pm 46 \mathrm{msec}$ (ranging from 15 to $160 \mathrm{msec}$ ) before the onset of the QRS complex (Table), and the duration was $151 \pm 85 \mathrm{msec}$. There was a difference in the duration and the precedence of the local electrogram between the VTs from a narrow site and VTs from a wide area, but they were not significant (duration 93 \pm 33 vs. $174 \pm 95 \mathrm{msec}, p>0.1$, precedence $30 \pm 12$ vs. $64 \pm 34 \mathrm{msec}, p<0.1$, respectively). The local electrogram at the site of origin showed fragmentation in 7 VTs and continuous activity in the other one VT in which rapid pacing re- 


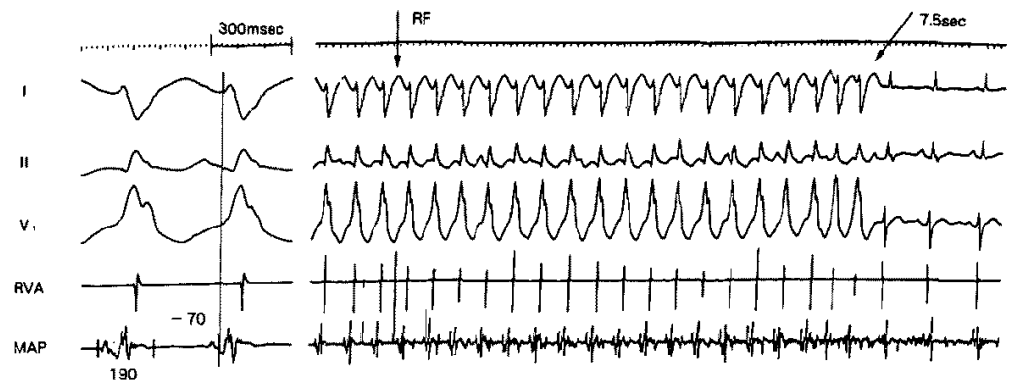

1000 msec

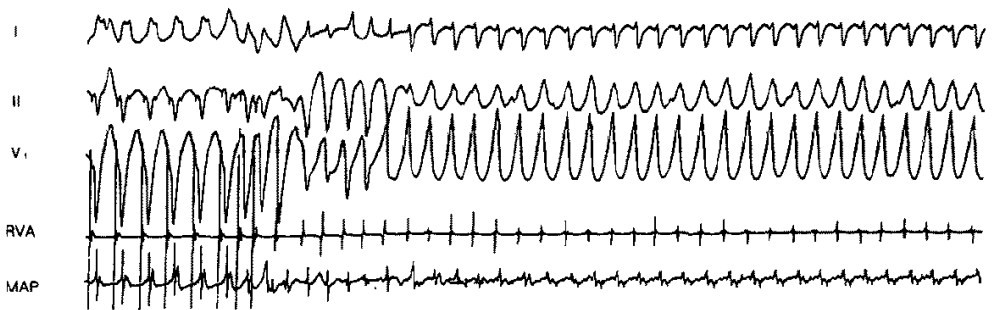

Figure 4. RF ablation and electrophysiologic study. This patient was a 68 -year-old man (Case 6). The slow conduction area was determined in the lateral wall of the left ventricle $(2.0 \times 3.0 \mathrm{~cm})$. The local electrogram at the ablation site showed fragmentation and the initiation was $70 \mathrm{msec}$ before onset of the QRS complex. An RF current was applicd to the central slow pathway of the VT, and the VT was terminated. However, a morphologically different VT was induced from the same area immediately after termination of the first VT. RVOT = outfiow tract of the right ventricle. Other abbreviations are the same as in Figures 1-3.

sulted in concealed entrainment. ${ }^{16)}$

Mapping finding versus effect of RF ablation: Two of 5 VTs with a narrow origin were eliminated by 2-3 effective RF applications (Figure 2). The other 3 V'Ts were not ablated by RF current, and they were associated with a postoperative state of congenital heart disease.

Five VTs originated from a wide arrhythmogenic area in 3 patients with idiopathic dilated cardiomyopathy. In 4 of the $5 \mathrm{VTs}$, RF current within the area terminated the VT, but VT with the same (Figure 3) or a slightly different QRS morphology (Figure 4) was induced. Two perpendicular linear RF lesions were then made within the area, and the 4 VTs became non-inducible. The method of two-dimensional RF ablation has been reported before. ${ }^{8,17)}$ Therefore, a relatively large number of effective RF applications (6.0 \pm 1.8 times) were required to ablate the VT from a wide arrhythmogenic area.

Two macroreentrant VTs (both revolving around a myotomy scar in clockwise and counterclockwise directions) (Case 7), were successfully ablated by linear $\mathrm{RF}$ application to the isthmus between the presumed upper edge of the vertical ventriculotomy scar and the pulmonary valve as reported previously. ${ }^{13)}$ 
Short-term results: With $2-8$ effective RF applications (4.6 \pm 2.1 times), 8 VTs became non-inducible immediately after RF ablation. Two weeks later, one of the 8 VTs was reinduced by programmed electrical stimulation, but was suppressed by antiarrhythmic drugs (disopyramide $300 \mathrm{mg} /$ day and propranolol 15 $\mathrm{mg}$ /day), which were ineffective before $\mathrm{RF}$ ablation. $\mathrm{RF}$ ablation was ineffective in the remaining $4 \mathrm{VTs}$ in 3 patients (Cases 2, 5, and 6), and these VTs were not terminated by RF application ( $7.0 \pm 3.5$ times). Further attempts were abandoned. Of these, VT was successfully ablated at the same sites by direct current catheter ablation in 3 VTs of 2 patients, ${ }^{18)}$ and an ICD was implanted in the other. Therefore, the acute success rate was $66.7 \%(8 / 12 \mathrm{VTs})$ or $62.5 \%(5 / 8$ patients).

Long-term results of $\mathbf{R F}$ ablation: After ablation, 5 successfully ablated patients had been followed-up for a period of 31.2 \pm 6.5 months (range 25-41 months). In one patient (Case 4), VT with a different QRS morphology occurred 27 months after RF ablation. The new VT originated from the free wall of the contralateral ventricle of the original VT.

Complications: No ST-T change was observed during or after ablation, and serum CK and CK-MB levels remained within the normal range after ablation. Two-dimensional echocardiography showed no abnormality immediately or 1-2 weeks after ablation.

\section{Discussion}

Mechanism of VT: Although reentry is the most common mechanism in ischemic sustained monomorphic VT, ${ }^{19,20)}$ other mechanisms may be involved in VT associated with non-ischemic underlying heart diseases. ${ }^{21}$

In this study, a reentrant mechanism was strongly suggested in 10 of 12 VTs from the observation of transient entrainment, two of which were macroreentry. The other 2 VTs (Cases 1 and 4) were induced during isoproterenol infusion, and no abnormal local electrogram was recorded during VT or in sinus rhythm. These findings suggest a non-reentrant mechanism of VT: automaticity or triggered activity. ${ }^{22,23}$

Endocardial mapping of VT origin: Unlike ischemic VT, the arrhythmogenic substrate of non-ischemic VT has not yet been fully elucidated. In this study, the site of VT origin was determined to be within a narrow site (less than $1.0 \times 1.0 \mathrm{~cm}$ ) in 5 VTs of 4 patients (Cases 1, 2, 5, and 8), while it was considered to have originated from a wide arrhythmogenic area (more than $2.0 \times 2.0 \mathrm{~cm}$ ) in 5 VTs of 3 patients (Cases 3, 4 and 6). This study did not include patients with an arrhythmogenic area ranging from $1.0 \times 1.0 \mathrm{~cm}$ to $2.0 \times 2.0 \mathrm{~cm}$, most likely because of the small number of patients. Two VTs were macroreentry revolving 
around a presumed operative scar in clockwise and counter clockwise directions. ${ }^{13)}$ Therefore, various arrhythmogenic substrates were observed in VT associated with non-ischemic underlying heart disease, and the indication and results of RF ablation should be examined on the basis of such arrhythmogenic substrates.

Mapping findings versus results of RF ablation: VTs originating from a narrow origin seem to be a good candidate for RF ablation, however, only 2 of the $5 \mathrm{VT}$ s from a narrow origin were successfully ablated in this study. The other 3 VTs were associated with postoperative congenital heart diseases, and were successfully treated later by direct current catheter ablation. ${ }^{18)}$ These findings suggest that VT in postoperative patients may originate deep in the myocardium $^{24)}$ or have pathological intervening tissues (fibrosis, calcification etc.) which limit the efficacy of RF ablation. Temperature monitoring of the catheter tip is helpful to assure adequate contact of the ablation catheter, although this was not employed in our study.

In VT from a wide arrhythmogenic area, RF current could terminate VT, but VT with the same or slightly different QRS morphology remained inducible. ${ }^{25)}$ We then delivered RF current in a systematic manner and made two perpendicular linear RF lesions to cover the wide arrhythmogenic area. ${ }^{17)}$ Such applications were expected to separate possible multiple slow pathways within the arrhythmogenic area, and 4 of the 5 VTs were successfully ablated.

Therefore, the efficacy of RF ablation seems to be strongly affected by the character of the arrhythmogenic substrate, although it is difficult to predetermine the effect of RF ablation before delivery of the RF current. However, once a VT is terminated by RF current, the VT may be ablated by additional RF applications. ${ }^{25)}$ In this study, all 8 VTs which had once been terminated by RF current, were successfully treated using RF ablation. Conversely, if VT cannot be terminated by an RF current, other therapeutic methods should be considered.

Long-term follow-up: VT with a different QRS morphology occurred in one patient with idiopathic dilated cardiomyopathy during the follow-up period. It is unlikely that the new VT was due to a proarrhythmic effect of prior RF lesions because the new VT originated from the free wall of the contralateral ventricle of the original VT. However, in patients with progressive underlying heart disease, repeated electrophysiologic studies may be necessary to predict the recurrence of VT. ICD may be ideal in such cases, although RF ablation seems to be useful for decreasing spontaneous VT attacks and maintaining battery longevity.

Limitations: The number of subjects in this study was small, and various underlying heart diseases were present. Each of the disorders is unique, thereby perhaps influencing the results of this study. However, the characteristic arrhythmogenic substrate was different even for the same underlying heart dis- 
ease. Studies using a larger number of patients are needed in order to evaluate the efficacy of RF ablation in non-ischemic VT with underlying heart disease. In this study, RF ablation was attempted only for drug refractory VT, although some patients had pleomorphic VTs. These patients may not be suitable candidates for RF ablation, and such bias in patient selection may have affected the results of this study.

Conclusions: RF catheter ablation was attempted in VT associated with nonischemic underlying heart disease, and the long-term results were examined. Termination using an RF current may be a hallmark to determine whether VT can be treated by RF ablation or not. The number of RF applications depends on the characteristics of the arrhythmogenic area. Two-dimensional RF ablation to cover the area was effective in VT having a wide arrhythmogenic area. Once VT has been ablated by RF ablation, excellent long-term efficacy can be expected.

\section{REFERENGES}

1. Klein LS, Shih HT, Hackett EK, Zipes DP, Miles WM. Radiofrequency catheter ablation of ventricular tachycardia in patients without structural heart disease. Circulation 1992; 85: 1666-74.

2. Aizawa $\mathbf{Y}$, Chinushi $M$, Naitoh $N$, et al. Catheter ablation with radiofrequency current of ventricular tachycardia originating from the right ventricle. Am Heart J 1993; 125: 1269-1275

3. Nakagawa H, Beckman KJ, McClelland JH, et al. Radiofrequency catheter ablation of idiopathic ventricular tachycardia by a Purkinje potential. Circulation 1993; 88: 2607-17.

4. Morady F, Harvey M, Kalbfleisch SJ, El-Atassi R, Calkins H, Langberg. IJ. Radiofrequency catheter ablation of ventricular tachycardia in patients with coronary artery disease. Circulation 1993; 87: 36372.

5. Gonska BD, Cao K, Schaumann A, Dorszewski A, Muhlen F, Kreuzer H. Catheter ablation of ventricular tachycardia in 136 patients with coronary artery disease: results and long-term follow-up. J Am Coll Cardiol 1994; 24: 1506-14.

6. Aizawa $\mathrm{Y}$, Niwano $\mathrm{S}$, Chinushi $\mathrm{M}$, et al. Incidence and mechanism of interruption of reentrant ventricular tachycardia with rapid ventricular pacing. Circulation 1992; 85: 589-95.

7. Aizawa M, Aizawa Y, Chinushi M, Takahashi K, Shibata A. Conductive property of the zone of slow conduction of reentrant ventricular tachycardia and its relation to pacing induced terminability. PACE $1994 ; 17: 46-55$.

8. Chinushi M, Aizawa Y, Kusano $\mathrm{Y}$, et al. Radiofrequency current catheter ablation for ventricular tachycardia. Jpn Circ J 1994; 58: 315-25.

9. Fontaine $\mathrm{G}$, Frank $\mathrm{R}$, Tonet $\mathrm{J}$, Grosgogeat $\mathrm{Y}$. Identification of a zone of slow conduction appropriate for VT ablation: theoretical and practical considerations. PACE 1989; 12: 262-267.

10. Morady F, Scheinman MM, Di Carlo LA, et al. Catheter ablation of ventricular tachycardia with intracardiac shocks: results in 33 patients. Circulation 1987; 75: 1037-1049.

11. Waldo AL, Henthorn RW, Plumb VJ, McLean WAH. Demonstration of the mechanism of transient entrainment and interruption of ventricular tachycardia with rapid atrial pacing. J Am Coll Cardiol 1984; 3: 422-30.

12. Aizawa $\mathrm{Y}$, Chinushi M, Kitazawa $\mathrm{H}$, et al. The spatial orientation of the reentrant circuit of idiopathic left ventricular tachycardia. Am J Cardiol 1995; 76:316-19.

13. Chinushi M, Aizawa Y, Kitazawa $\mathbf{H}$, Kusano Y, Washizuka $T$, Shibata A. Successful radiofrequency catheter ablation for macroreentrant VT in a patient with tetralogy of Fallot after correcuive surgery. PACE 1995; 18: 1713-16. 
14. Stevenson WG, Weiss JN, Wiener 1, et al. Fractionated endocardial electrograms are associated with slow conduction in humans; evidence from pace-mapping. J Am Coll Cardiol 1989; 13: 369-76.

15. Chinushi M, Aizawa Y, Kusano Y, Washizuka T, Shibata A. Evidence for slow conduction areas during pacing in patients with sinus rhythm, and their relation to the site of VT origin. Jpn Heart $J$ 1994; 35: 113

16. Morady F, Frank R, Kou WH, et al. Identification and catheter ablation of a zone of slow conduction in the reentrant circuit of ventricular tachycardia in humans. J Am Coll Cardiol 1988; 11: 775-82.

17. Chinushi M, Aizawa $\mathrm{Y}$, Naitoh N, Kusano Y, Kitazawa H, Shibata A. Attempt at catheter ablation with radiofrequency current for VT with extending arrhythmogenic area; cross-sectional ablation. PACE 1994; 17: 831 (abstract).

18. Niwano S, Aizawa Y, Koike T, Shibata A. Successful transeptal catheter electrical ablation of sustained ventricular tachycardia of possible intramuscular origin. Jpn Heart J 1992; 33: 403-7.

19. Josephson ME, Horowitz LN, Farshidi A, Kastor JA. Recurrent sustained ventricular tachycardia. I. Mechanisms. Circulation 1978; 57: 431-40.

20. Stevenson WG, Weiss J, Wiener I, Wohlgelernter D, Yeatman L. Localization of slow conduction in a ventricular tachycardia circuit; implications for catheter ablation. Am Heart J 1987; 114: 1253-8.

21. Aizawa $\mathrm{X}$, Naitoh $\mathbf{N}$, Kitazawa $\mathrm{H}$, et al. Incidence of reentry with excitable gap in sustained ventricular tachycardia unassociated with coronary artery disease. Am J Cardiol 1994; 72: 916-21.

22. Lerman BB. Response of nonreentrant catecholamine-mediated ventricular tachycardia to endogenous adenosine and acetylcholine. Circulation 1993; 87: 382-90.

23. Vlay SC. Catecholamine-sensitive ventricular tachycardia. Am Heart J 1987; 114: 455-61.

24. Chinushi M, Aizawa $Y$, Masani F, Obata A, Shibata A. Possible intramural site of reentrant circuit in ventricular tachycardia of nonischemic cause. Pre and intraoperative mapping study. Jpn Heart J 1992; 33: $505-12$.

25. Aizawa Y, Chinushi M, Naitoh N, et al. Catheter ablation of ventricular tachycardia with radiofrequency currents with special reference to the termination and minor morphological change of reinduced ventricular tachycardia. Am J Cardiol 1995; 76: 574-579. 